\title{
Firing Pattern Characteristics of Tonically Active Neurons in Rat Striatum: Context Dependent or Species Divergent?
}

\author{
Liora Benhamou, ${ }^{1}$ Orli Kehat, ${ }^{2}$ and Dana Cohen ${ }^{1}$ \\ ${ }^{1}$ The Leslie and Susan Gonda Multidisciplinary Brain Research Center, Bar-Ilan University, Ramat-Gan 52900, Israel, and ${ }^{2}$ The Institute for the Study of \\ Affective Neuroscience, University of Haifa, Haifa 31905, Israel
}

\begin{abstract}
Tonically active neurons (TANs) - presumably, striatal cholinergic interneurons- exert a strong influence on striatal information processing. Primate studies often describe a characteristic TAN response comprising suppressed activity followed by rebound firing that occasionally is preceded by a brief activation. By contrast, studies in behaving rats report pronounced excitation during movement. These differences in firing patterns may be due to variations in behavioral conditions or could stem from the fact that TANs in rodents use different neuronal mechanisms. If similar/different task conditions yield similar/different activity patterns, then the two species may share neuronal mechanisms; however, if similar task conditions yield different activity patterns, then the two species use different neuronal mechanisms. To evaluate these possibilities, we recorded TAN activity in the ventral and dorsolateral striatal regions in rats performing a simple instrumental task similar in concept to one used in primate studies. We demonstrate that TAN activity is substantially influenced by event context; yet, under identical task conditions, primate and rat TANs display similar activity patterns, whereas under different conditions they do not. Our results suggest that the observed differences in firing patterns likely reflect dissimilarities in task attributes rather than species-dependent neuronal mechanisms and call for re-evaluation of the excitatory response in primate research.
\end{abstract}

Key words: chronic recording; electrophysiology; instrumental task; operant conditioning; reward

\section{Introduction}

The striatum is composed of a majority (95\%) of projection neurons_-the medium spiny neurons (MSNs) - whose activity is shaped by a diversity of relatively rare interneurons (Koós and Tepper, 1999; Witten et al., 2010). Most of the identified interneurons are GABAergic with the exception of the cholinergic tonically active neurons (TANs) (Inokawa et al., 2010), which are considered to be key mediators of dopamine-dependent striatal plasticity and learning (Wang et al., 2006). Studies of TAN activity in classical and instrumental conditioning experiments often describe a characteristic response comprising suppressed activity followed by rebound firing that occasionally is preceded by a brief activation (Kimura et al., 1984; Aosaki et al., 1994). Only a relatively small number of primate studies have found robust TAN activation, for example, during conditioned reward (Kimura, 1992), free reward, or self-paced movement (Lee et al., 2006), and following reward omission (Apicella et al., 2009). Consequently, the pause response has become the hallmark of TAN activity in primates (Goldberg and Reynolds, 2011), and is thought to pro-

\footnotetext{
Received April 30, 2013; revised Nov. 15, 2013; accepted Nov. 21, 2013.

Author contributions: L.B., 0.K., and D.C. designed research; L.B. and 0.K. performed research; L.B. and D.C. analyzed data; L.B. and D.C. wrote the paper.

This study was supported in part by REALNET Grant FP7-ICT270434 from the European Commission and by Israel Science Foundation Grant 861/06.

The authors declare no competing financial interests.

Correspondence should be addressed to Dana Cohen, The Leslie and Susan Gonda Multidisciplinary Brain Research Center, Bar-Ilan University, Ramat-Gan 52900, Israel. E-mail: danacoh@gmail.com.

DOI:10.1523/JNEUROSCI.1798-13.2014

Copyright $\odot 2014$ the authors $\quad 0270-6474 / 14 / 342299-06 \$ 15.00 / 0$
}

mote the saliency of a concomitant change in dopamine neuronal activity during bursting activity and possibly pauses (Cragg, 2006). In contrast to this typical pause response, rat TANs displayed robust excitation during movement performed in the context of a cued two-choice alternative instrumental task (Yarom and Cohen, 2011). The activity of primate TANs has never been studied during a similar task.

Recent cellular studies have shed new light on the complex regulatory influence TANs exert on other striatal neurons, particularly through their excitation (Schulz and Reynolds, 2013). Thalamic input to the striatum engages cholinergic interneurons, which, by activating muscarinic receptors, gate the corticostriatal control of striatopallidal and striatonigral networks (Ding et al., 2010); TAN activation causes dopamine release in the striatum through activation of nicotinic receptors independently of midbrain neuronal activity (Cachope et al., 2012; Threlfell et al., 2012), and TANs activate a GABAergic subpopulation, which in turn leads to widespread recurrent inhibition of the TANs (Sullivan et al., 2008) and the MSNs (English et al., 2012).

On one hand, the prominent component of TAN activity in primates is considered to be the pause, whereas the rodent literature covering both cellular and behavioral studies emphasizes the excitatory component of TAN activity. This mismatch could be the outcome of divergent neural mechanisms across species, or alternatively, it could be that task conditions used in rodent and primate studies were different in essence.

To test these possibilities, we recorded and analyzed TAN activity in the ventral striatum (VS) and dorsolateral striatum 

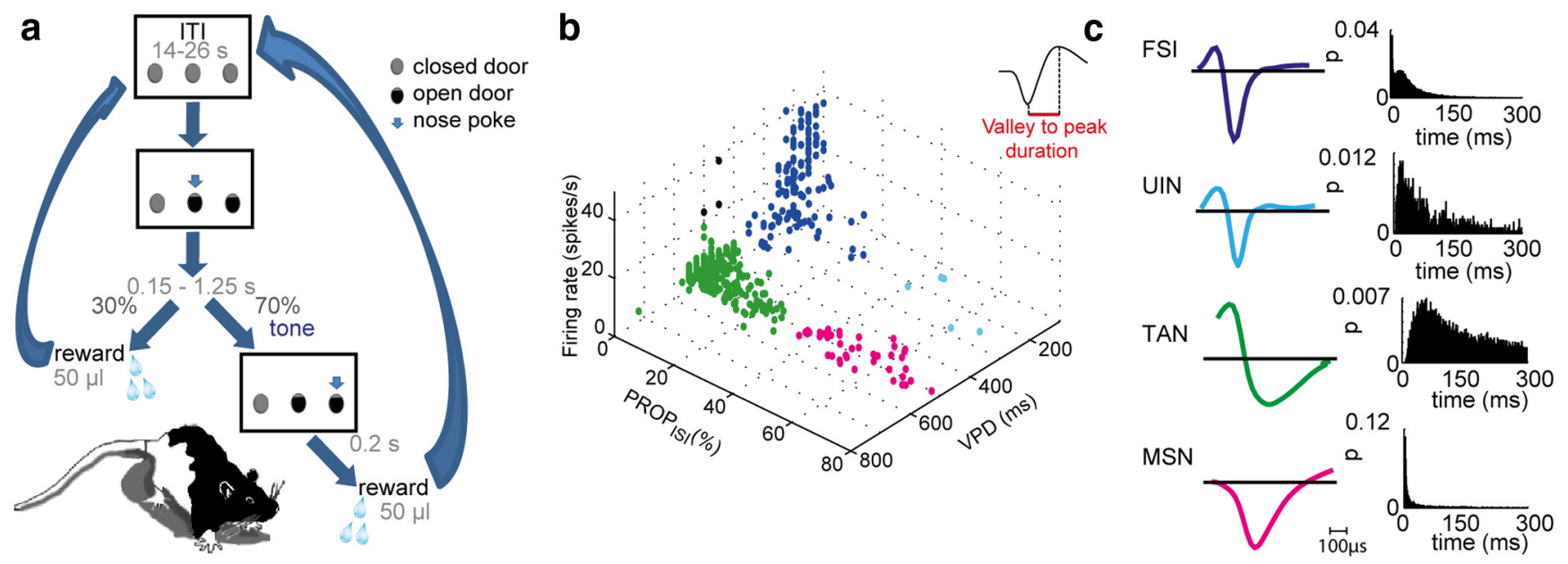

Figure 1. Behavioral paradigm and neuronal classification. $\boldsymbol{a}$, Behavioral paradigm schematic (see text for details). $\boldsymbol{b}, 3 \mathrm{D}$ scatter plot of waveform parameters and firing characteristics of sorted single neurons reveal four distinct clusters. Inset, VPD definition. c, Representative single-neuron examples of waveforms and ISI distributions of the four groups.

(DLS) in well trained rats performing a simple instrumental task. We show that, similar to primates, rat TANs pause in response to a GO signal and to reward delivery; however, deviations in task conditions yield a broad repertoire of activity patterns. Our results demonstrate that TAN activity is substantially influenced by event context, and support the possibility that the observed differences in firing patterns likely reflect dissimilarities in task attributes rather than species-divergent neuronal mechanisms.

\section{Materials and Methods}

All procedures were approved by the Bar-Ilan University Animal Care and Use Committee and performed in accordance with the National Institutes of Health guidelines.

\section{Behavioral paradigm}

Pretraining. Adult male Long-Evans rats were pretrained to nose-poke a hole and wait for a tone (frequency: $12 \mathrm{kHz}$; duration: $200 \mu \mathrm{s}$ ) until they learned to wait for $550-700 \mathrm{~ms}$ in $85 \%$ of the trials. Upon reaching the criterion, rats were removed from the water deprivation protocol and underwent surgery. After recovery, rats were retrained for $2-5 \mathrm{~d}$ on the pretraining task after which training on the instrumental task began.

Instrumental task. The behavioral paradigm is illustrated in Figure $1 a$. At trial onset, the central and right doors were opened, and the rats had to nose-poke the central hole until a tone (frequency: $7 \mathrm{kHz}$; duration: 200 $\mu \mathrm{s})$ serving as a GO signal was played after a randomized waiting period of 150-1250 ms. Rats had then to nose-poke the right hole to obtain $50 \mu \mathrm{l}$ of water reward delivered after $200 \mathrm{~ms}$ (expected reward). Following premature head retraction, the doors were immediately closed, and the trial was repeated. Intertrial intervals (ITIs) ranged from 14 to $26 \mathrm{~s}$. Infrared source detectors placed behind each door time stamped the entrance of the rat into and retraction from the hole.

Unexpected reward. In $30 \%$ of the trials occurring within sessions that followed performance stabilization, the water reward was unexpectedly delivered in the central hole instead of the tone. Reaction times (i.e., the times elapsed between the playing of the tone and head retraction from the hole) during the performance of regular trials before and after the introduction of unexpected reward remained unchanged $(0.65 \pm 0.32$ and $0.61 \pm 0.23 \mathrm{~s}$, respectively; $p=0.489$ ), suggesting that the rats were tuned to the GO signal and not reward delivery.

\section{Surgical procedure}

The surgical and histological procedures have been described at length previously (Yarom and Cohen, 2011). In this study, electrodes were bilaterally implanted in VS [anteroposterior (AP): $-1.5 \mathrm{~mm}$; mediolateral (ML): $1.8 \mathrm{~mm}$; dorsoventral (DV): $6.8 \mathrm{~mm}$ ] and DLS (AP: $0.5 \mathrm{~mm}$; ML: $3.9 \mathrm{~mm}$; DV: $3.9 \mathrm{~mm}$ ) in all rats.

\section{Data collection}

Neural activity was amplified, bandpass filtered at $150-8000 \mathrm{~Hz}$, and continuously sampled at $40 \mathrm{kHz}$ using a multichannel acquisition processor system (MAP system; Plexon Inc). Off-line sorting was performed on all recorded units. If a neuron was recorded for $>1 \mathrm{~d}$, only the first recording day was included in the analysis. The data were then transferred to MATLAB software (R2010b; MathWorks) for further analysis.

\section{Neuronal classification}

Immunochemical identification of cell types in chronic extracellular recordings of behaving animals is technically challenging in particular when recording sessions spread over weeks. Therefore, the recorded neurons were classified into cell types by using the following waveform and firing features measured in each session on data recorded before task commencement: valley to peak duration (VPD), proportion of interspike intervals (ISIs) exceeding $2 \mathrm{~s}\left(\mathrm{PROP}_{\mathrm{ISI}}\right)$ and firing rate $(\mathrm{FR})$, which enabled reliable cell classification in mice (Yamin et al., 2013). As in the mouse, four distinct clusters emerged, with each corresponding to a different cell type (Fig. 1b). Representative waveforms and ISI distributions of each cell type are shown in Figure 1c. Population averages of all features per cell type were as follows: MSNs (magenta; VPD $=497.0 \pm 37.5 ; \mathrm{PROP}_{\mathrm{ISI}}>35 \%$; FR $=1.0 \pm 0.8$ ); TANs (green; $\mathrm{VPD}=498.4 \pm 42.8 ; \mathrm{PROP}_{\mathrm{ISI}}<35 \%$; $\mathrm{FR}=4.4 \pm 1.6$ ); fast-spiking interneurons (FSIs; blue; VPD $=201.5 \pm 59.3$; $\mathrm{PROP}_{\text {ISI }}<35 \%$; $\mathrm{FR}=16.5 \pm 11.3$ ); and unidentified interneurons (UINs; light blue; $\left.\mathrm{VPD}=215.0 \pm 65.2 ; \mathrm{PROP}_{\mathrm{ISI}}>35 \% ; \mathrm{FR}=1.1 \pm 0.8\right)$. Of the 588 recorded neurons (VS: 281 neurons; DLS: 307 neurons), we obtained 42 MSNs (VS: 16 MSNs; DLS: 26 MSNs), 398 TANs (VS: 230 TANs; DLS: 168 TANs), 140 FSIs (VS: 31 FSIs; DLS: 109 FSIs), and 5 UINs (VS: 3 UINs; DLS: 2 UINs).

\section{Data analysis}

Neural responses to behavioral events were first characterized by their perievent time histograms (PETHs) calculated in $10 \mathrm{~ms}$ bins in a -300 to $700 \mathrm{~ms}$ time window around the event. The baseline rate was subtracted, and PETHs were $z$-scored. Note that the $z$-score values do not automatically imply discharge rate fluctuations compared with baseline rate but rather reflect the neuronal pattern around the event under investigation.

Clustering of event-related activity groups. TAN responses were clustered into groups as thoroughly described by Adler et al. (2012). Here, the $\mathrm{K}$-means algorithm was used to classify the activity into distinct groups based on the first two principal components calculated for the following event-related activities: tone, expected reward, unexpected reward, reward omission, and trial onset.

Significant responses. The relevant time window and delay for determining event-related responses was selected based on the average response of each clustered group. A neuron was considered to be 
a

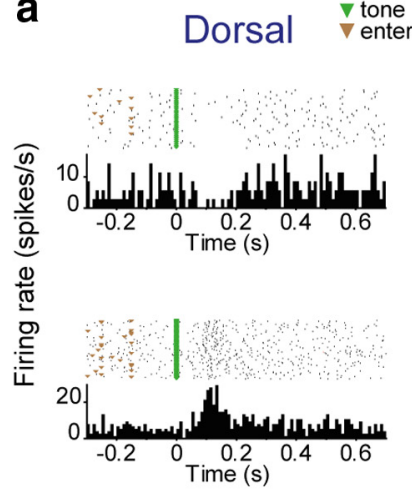

b

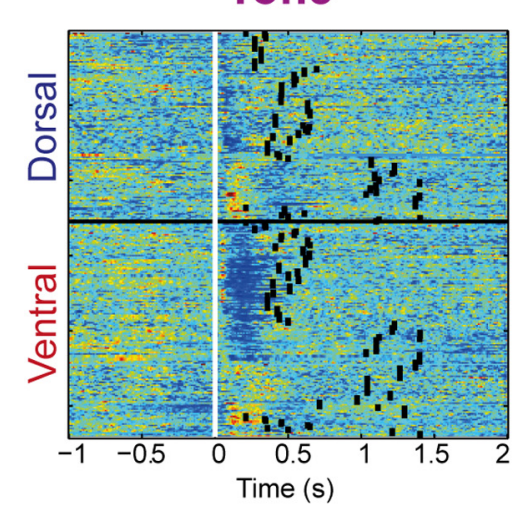

C

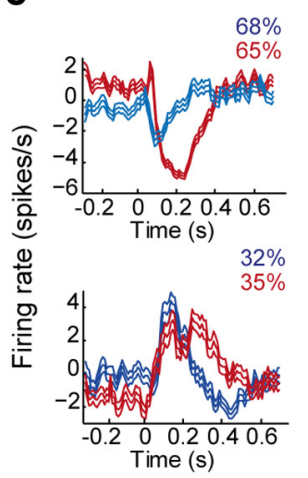

d Movement initiation

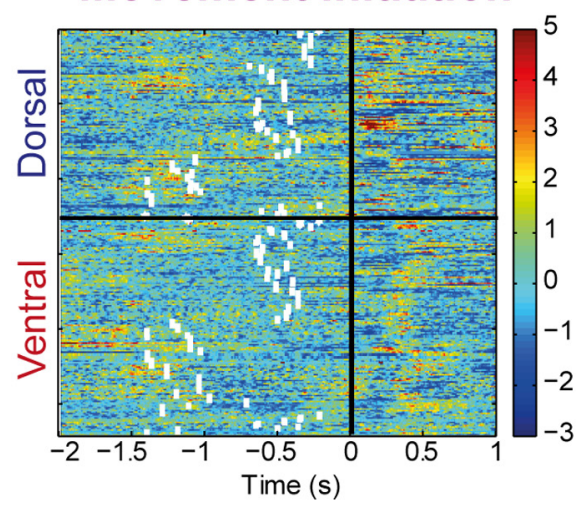

Figure 2. TAN responses to the tone. $\boldsymbol{a}$, Single-neuron examples of the different firing patterns observed in the DLS (left) and VS (right). $\boldsymbol{b}$, Each row is the color-coded z-scored PETH of a single TAN aligned to the tone (white squares). All the recorded neurons are shown from top to bottom as classified by the K-means algorithm. Black squares denote median movement initiation. $\boldsymbol{c}$, The average responses to the tone of all neurons within a cluster are shown for DLS (blue) and VS (red). $\boldsymbol{d}$, Same as $\boldsymbol{b}$ with activity aligned to movement initiation.

event-related if its activity distribution over trials during the selected time window following the event significantly differed from its baseline activity distribution (Wilcoxon rank sum test, $p<0.01$ ). The widths of the responses were calculated at half-height and compared between groups (Wilcoxon rank sum test, $p<0.05$ ). All the data are shown as the mean \pm SD.

\section{Results}

We recorded neuronal activity in the DLS and the VS of eight rats performing a simple instrumental task. To correctly perform the task, rats had to nose-poke a central hole and wait for an auditory GO signal instructing them to nose-poke the right hole (Fig. 1a). Within a session, the rats performed an average of $97 \pm 5$ correct trials and $7 \pm 0.5 \%$ of error trials in which they prematurely retracted their head from the central hole. The average time between trial onset and central hole nose poke was $1.26 \pm 2.76 \mathrm{~s}$, and the average movement time to the side hole was $0.48 \pm 0.5 \mathrm{~s}$. The recorded neurons were classified into cell types based on their properties (see Materials and Methods), and those identified as TANs (VS: $n=230$ TANs; DLS: $n=168$ TANs) were taken for further analysis. Identical TAN responses were observed during spatially irrelevant events (i.e., all the results described below for right hole training resembled those obtained for left hole training).

First, we examined TAN responses (VS: $n=167$ TANs; DLS: $n=129$ TANs) to the GO signal (tone). TANs in both regions tended to either pause or increase their firing rate following the tone (Fig. 2a). Classification of the normalized ( $z$-score) tone response profiles of all TANs by the K-means algorithm (see Materials and Methods) partitioned the data into two groups

(Fig. 2b). The large group jointly paused activity $(65 \%$ and $68 \%$ in VS and DLS, respectively) whereas the small group enhanced its activity (35\% and $32 \%$ in VS and DLS, respectively) in response to the tone (Fig. 2c). Both response types were attenuated when neuronal activity was aligned to movement initiation; $z$-scores aligned to the tone $(0.85 \pm 0.14)$ were significantly higher than those aligned to movement onset $(0.76 \pm 0.19 ; p=3.79 \times$ $\left.10^{-14}\right)$, thus demonstrating that these responses were tone rather than movement preparation related (Fig. $2 d$ ). Specifically, $49 \%$ of the TANs in the VS and $61 \%$ in the DLS significantly altered their firing rate following the tone, and the same ratio as in the whole population of pausing versus excited TANs was grossly maintained (pause: $76 \%$ and 70\%; excitation: $24 \%$ and $30 \%$, in VS and DLS, respectively). The widths of the two response types were broader in the VS (pause: $143.4 \pm 60.7$ ms; excitation: $169.3 \pm 78.3 \mathrm{~ms}$ ) than in the DLS (pause: $65.5 \pm 91.6 \mathrm{~ms}$; excitation: $115.8 \pm 52.7 \mathrm{~ms}$ ); however, the difference was significant only for the pause ( $p=0.0008$ and $p=0.055$ for the pause and excitation, respectively).

The TAN responses to a reward delivered at the end of each trial (expected reward) revealed a complex temporal relation between the pause and excitation within the same neuron. In this case, classification of the normalized reward response profiles of all the TANs partitioned the data into two groups in the VS and three groups in the DLS (Fig. $3 a$ ). A close examination of the groups' response averages showed that all of them included a pause component at varying time delays relative to reward delivery, whereas excitation occasionally preceded or followed the pause (Fig. $3 b$ ). Examples of single-neuron activity representative of each group are shown in Figure $3 c$. A significantly larger proportion of DLS TANs (50\%) responded to the expected reward compared with VS TANs $\left(37.5 \%\right.$; $\chi^{2}$ test, $p=$ $0.03)$, of which the vast majority of the neurons paused their activity, although only $8 \%$ of them (3\% in VS; $5 \%$ in DLS) displayed excitation without a significant pause. Overall, TANs in the VS and DLS primarily displayed a pause response that occasionally was preceded or followed by enhanced activation.

Analysis of the TAN responses to a reward delivered unexpectedly in the center hole while the animals were waiting for the tone (unexpected reward; see Materials and Methods) revealed an entirely different scenario. The classification algorithm partitioned the data into three groups (Fig. $3 d$ ). The TANs in two of these groups (a total of $50 \%$ and $56 \%$, in VS and DLS, respectively) increased their firing rate for different durations, whereas in the third group ( $50 \%$ and $44 \%$, in VS and DLS, respectively) the TANs paused their activity (Fig. $3 e$ ). Examples of single neurons representative of the different groups are shown in Figure $3 f$. Similar percentages of TANs in the VS (39\%) and DLS (37\%) significantly altered the firing rate in response to unexpected reward. However, much to our surprise, the number of TANs that substantially increased their firing rate in both regions (VS: 74\%; 
a Expected reward

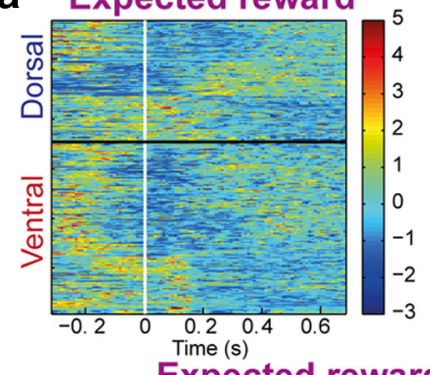

d Unexpected reward

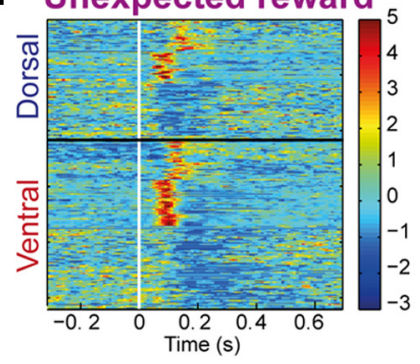

g

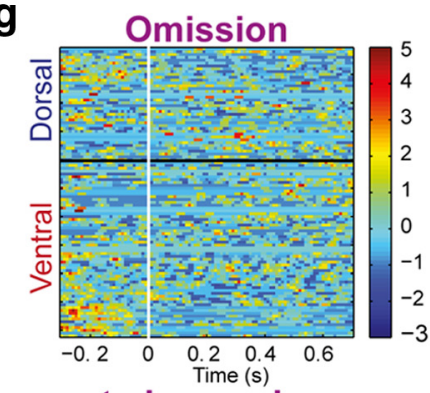

Unexpected reward center hole

b side hole
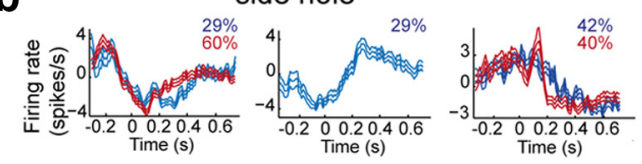

C
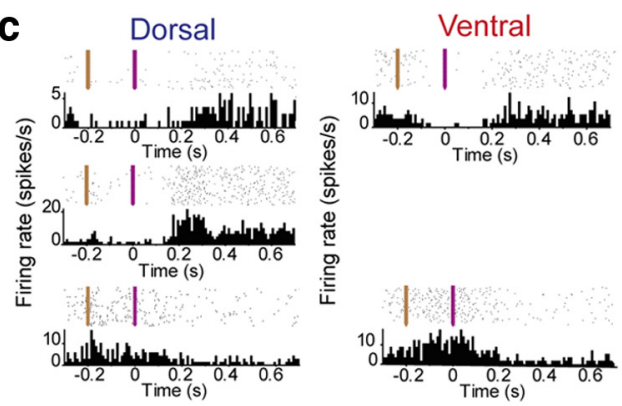

e

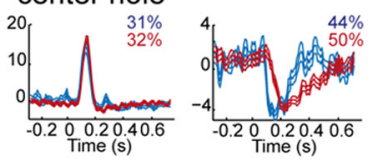

$\checkmark$ enter hole
$\checkmark$ reward

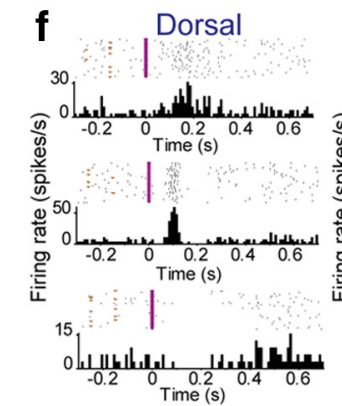

Figure 3. TAN responses to different reward contexts. $\boldsymbol{a}$, Each row is the color-coded $z$-scored PETH of a single TAN aligned to the expected reward (white). All the recorded neurons are shown from top to bottom as classified by the K-means algorithm. $\boldsymbol{b}$, The average responses to expected reward of all neurons within a cluster are shown for DLS (blue) and VS (red). $\boldsymbol{c}$, Single-neuron examples of the observed firing patterns in DLS (left) and VS (right) after expected reward. $\boldsymbol{d}-\boldsymbol{f}$, Same as $\boldsymbol{a}$ - c for reward delivered unexpectedly in the center hole. $\boldsymbol{g}$, Same as $\boldsymbol{a}$ for reward omission. PETHs were aligned to the expected time of reward delivery.

DLS: 75\%) significantly exceeded that of the TANs that paused their activity (VS: $26 \%$; DLS: $25 \% ; \chi^{2}$ test, $p=0.001$ and $p=$ 0.025 for VS and DLS regions, respectively). These results run counter to the observed responses to the tone and the expected reward, where the majority of neurons paused activity.

To test whether the TAN responses to unexpected reward could be explained by the element of surprise, we analyzed in a separate group of animals $(n=4)$ TAN ( $n=63$ and 39, in VS and DLS, respectively) responses to uncued omission of the expected reward in $\sim 10 \%$ of the trials. A negligible number of neurons $(0$ and 5\% in VS and DLS, respectively) significantly altered their firing rate in response to reward omission, thus suggesting that TAN responses did not correlate with the element of surprise (Fig. $3 g$ ). TAN responses to all other events remained the same as previously described.

The most striking responses of the TANs occurred when the door blocking the center hole opened and signaled the animal that it could initiate a trial by nose-poking the center hole (trial onset; Fig. 4a). The observed responses to trial onset were severely attenuated when aligned to the animals' arrival at the center hole; $z$-scores aligned to trial onset $(0.93 \pm 0.13)$ were significantly higher than those aligned to center hole arrival time $(0.76 \pm 0.20$; $\left.p=1.2 \times 10^{-36}\right)$, thus demonstrating that these responses were locked to the door opening and not to the movement into the hole (Fig. 4b). As detected by the K-means algorithm, the VS displayed two activity profiles, an excitation and a pause, whereas the DLS displayed three activity profiles, two of excitation and the third of a pause (Fig. 4c). A representative example of each group is shown in Figure $4 d$. Note the robust excitation that reached an exceptionally high firing rate of 40 spikes/s in the VS and DLS.
The percentages of excitation versus a pause in the VS were $66 \%$ and $34 \%$, respectively. The ratio of percentages was accentuated in the DLS, where the excitation and the pause response group sizes were $74 \%$ and $26 \%$, respectively. The two excitatory DLS groups displayed different response durations that did not reach significance-brief activation ( $42 \%$; $95 \pm 47 \mathrm{~ms})$ and long activation $(32 \% ; 120 \pm 61 \mathrm{~ms} ; p=0.08)$. Overall, $67 \%$ of TANs $(112$ of 167 TANs) in the VS and 75\% of TANs (97 of 129 TANs) in the DLS significantly altered their firing rate following trial onset, which was substantially more than TAN responses to the other events ( $\chi^{2}$ test, $p<0.01$ and $p<0.05$ in VS and DLS, respectively). The ratio of excitation versus pause in both striatal regions (pause: $20 \%$ and $28 \%$; excitation: $80 \%$ and $72 \%$, in VS and DLS, respectively) was similar to that observed during the unexpected reward, and was completely contrary to the expected reward and the GO signal responses.

\section{Discussion}

In the present study, we demonstrated that, as reported in primate studies during a simple instrumental task, rat TANs predominantly paused in response to a GO signal and to expected reward delivery. By contrast, during trial onset and unexpected reward delivery, the TANs substantially increased their firing rate without displaying a preceding pause; furthermore, rat TANs did not respond to unexpected omission of reward. VS and DLS TANs displayed similar firing patterns and a similar percentage of event-responsive neurons, except for expected reward delivery during which substantially more DLS TANs paused compared with VS TANs. When a more complex task such as a two-choice alternative association task was used, substantial inter-regional 
a Trial onset
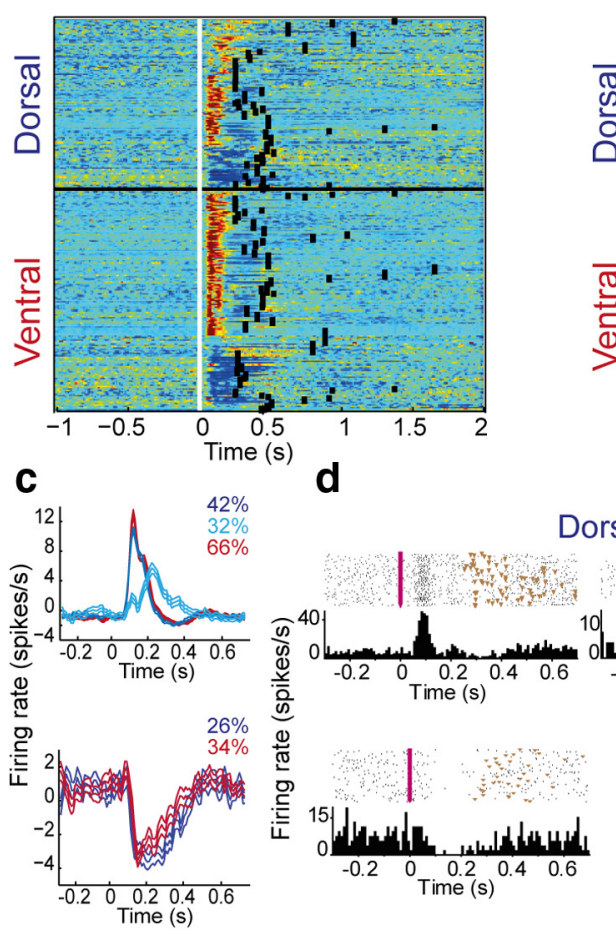

b Nose poke center hole

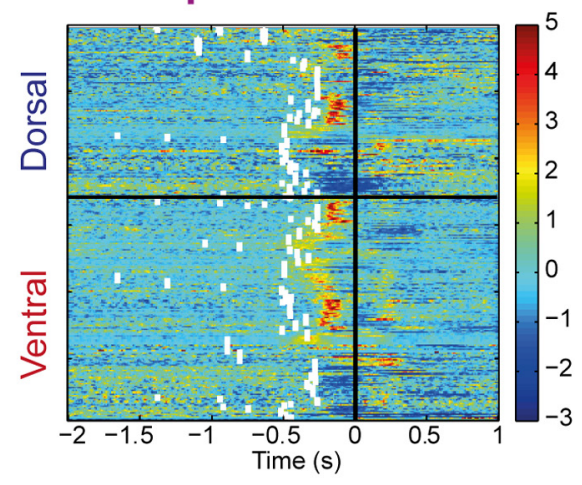

Figure 4. TAN responses to trial onset. $\boldsymbol{a}$, Each row is the color-coded $z$-scored PETH of a single TAN aligned to trial onset (white squares). All the recorded neurons are shown from top to bottom as classified by the K-means algorithm. Black squares denote median nose-poking of the central hole. $\boldsymbol{b}$, Same as $\boldsymbol{a}$ but aligned to nose-poking of the central hole. $\boldsymbol{c}$, The average responses to trial onset of all neurons within a cluster are shown for DLS (blue) and VS (red). $\boldsymbol{d}$, Single-neuron examples of the observed firing patterns in DLS (left) and VS (right) after trial onset.

differences appeared (Yarom and Cohen, 2011), thus suggesting that task difficulty influences the allocated resources, thus emphasizing the difference between DLS and VS function.

The fact that during conditioned cues such as a GO signal and expected reward delivery rat TANs display activity patterns resembling those observed in primates during the same events provides supporting evidence for the assumption that rat and primate TANs share similar neuronal mechanisms. By contrast, the fundamental difference in TAN responses to task events such as unexpected reward delivery and trial onset casts doubt on the validity of this assumption and instead raises the possibility that TANs in the two species use different neuronal mechanisms. Nonetheless, careful examination of the exact experimental conditions during which activity was recorded in the two species reveals task dissimilarities that may account for the broad repertoire of activity patterns displayed by rat TANs, thus supporting the possibility that rat and primate TANs share similar neuronal mechanisms.

First, most primate studies describe TAN responses to cues signaling reward delivery or instructing the monkey to perform a required movement. Such cues are conceptually similar to the tone instructing rats to move rightward, but they do not correspond to the trial onset cue in our paradigm. The fundamental difference between these cues and the trial onset cue is that during the long ITIs (14-25 s) rats have time to engage in different actions. Once the center door opens, they immediately abort their action and nosepoke the central door. By contrast, in primate studies the ITIs are shorter (3-8s) and the monkeys are constantly alert to cue appearance (Kimura, 1992; Joshua et al., 2008; Apicella et al., 2011). Hence, the strong excitatory TAN response may indicate either the imme- diate need to abort an ongoing action or may redirect the attention of the rat to the task and trial initiation (Ding et al., 2010).

Second, delivering a reward outside of the instrumental task resulted in most studies in the typical pause response (Kimura et al., 1984; Kimura, 1992; Ravel et al., 2001) with the exception of one study that found robust phasic activation (Lee et al., 2006) and another that showed that the TANs did not respond to uncued random reward (Aosaki et al., 1994). In our experiments, the rats were free to move in the behavioral box, making it impossible to deliver a surprising reward during the intertrial interval without confounding body movements. Hence, reward was randomly delivered by surprise after trial onset as the animal was waiting for the GO signal. When a similar paradigm was used in primates, more TANs responded compared with the expected reward condition; however, all TANs displayed a pause (Ravel et al., 2001). Close examination of task conditions revealed a small yet important difference in experimental design; in the primate task, the monkey was required to continue and press a bar, whereas the rat was not required to maintain its posture. Plausible interpretations would be that (1) in the rat task the reward marked the end of a trial, whereas in the primate task it did not; and (2) the rats had to instantly withhold their planned whole-body movement toward the right hole, whereas the monkey only had to withhold an arm, which requires fewer resources.

Third, a negligible number of rat TANs responded to uncued omission of expected reward. Similarly, during block trials of an instrumental task in which a reward cue was presented without delivering a reward, primate TANs did not respond to the cue (Kimura, 1992). In other studies of reward omission during classical conditioning, primate TANs displayed a pause (Aosaki et al., 1994); however, it was the excitatory rebound phase of the pattern that was enhanced during a probabilistic version of this conditioning (Apicella et al., 2009). During the performance of the instrumental version of the task, TANs displayed an attenuated pause response (Morris et al., 2004), which increased when reward omission was cued (Joshua et al., 2008). Overall, when reward is omitted, primate TANs seem to display a broad response repertoire, depending on task conditions. These diverse responses could reflect variations in the attention of the animals, the relevance of reward omission to the animal, or the outcome of different reward expectations.

In addition to showing the similarities in primate and rat TAN activity patterns that occurred when the cues and events had similar meaning, our research also highlights fundamental differences in behavioral paradigms typically used by each species, which may underlie the observed differences. It should be noted that the TAN subpopulation, presumed to be the cholinergic interneurons, may be contaminated by other cell types that display tonic firing and a wide waveform like the low-threshold spiking interneurons (Beatty et al., 2012). The level of contamination is influenced by the prevalence of such neurons in rats 
relative to primates and the recording method. Nonetheless, our results call for re-evaluation of an excitatory component in primate TAN activity and for further testing of whether the observed interspecies differences could be attributed to diverse experimental conditions by designing behavioral paradigms that could be compared in essence across species. Of special interest is the finding of primate behavioral paradigms that resemble the conditions and/or meaning of trial onset as in the rat paradigm. If confirmed, then performing comparative studies would facilitate the characterization of TAN contribution to behavior, thus promoting comprehensive understanding of basal ganglia function.

\section{References}

Adler A, Katabi S, Finkes I, Israel Z, Prut Y, Bergman H (2012) Temporal convergence of dynamic cell assemblies in the striato-pallidal network. J Neurosci 32:2473-2484. CrossRef Medline

Aosaki T, Tsubokawa H, Ishida A, Watanabe K, Graybiel AM, Kimura M (1994) Responses of tonically active neurons in the primate's striatum undergo systematic changes during behavioral sensorimotor conditioning. J Neurosci 14:3969-3984. Medline

Apicella P, Deffains M, Ravel S, Legallet E (2009) Tonically active neurons in the striatum differentiate between delivery and omission of expected reward in a probabilistic task context. Eur J Neurosci 30:515-526. CrossRef Medline

Apicella P, Ravel S, Deffains M, Legallet E (2011) The role of striatal tonically active neurons in reward prediction error signaling during instrumental task performance. J Neurosci 31:1507-1515. CrossRef Medline

Beatty JA, Sullivan MA, Morikawa H, Wilson CJ (2012) Complex autonomous firing patterns of low-threshold spike interneuros. J Neurophysiol 108:771-781. CrossRef Medline

Cachope R, Mateo Y, Mathur BN, Irving J, Wang HL, Morales M, Lovinger DM, Cheer JF (2012) Selective activation of cholinergic interneurons enhances accumbal phasic dopamine release: setting the tone for reward processing. Cell Rep 2:33-41. CrossRef Medline

Cragg SJ (2006) Meaningful silences: how dopamine listens to the ACh pause. Trends Neurosci 29:125-131. CrossRef Medline

Ding JB, Guzman JN, Peterson JD, Goldberg JA, Surmeier DJ (2010) Thalamic gating of corticostriatal signaling by cholinergic interneurons. Neuron 67:294-307. CrossRef Medline

English DF, Ibanez-Sandoval O, Stark E, Tecuapetla F, Buzsáki G, Deisseroth K, Tepper JM, Koos T (2012) GABAergic circuits mediate the reinforcement-related signals of striatal cholinergic interneurons. Nat Neurosci 15:123-130. CrossRef Medline

Goldberg JA, Reynolds JN (2011) Spontaneous firing and evoked pauses in the tonically active cholinergic interneurons of the striatum. Neuroscience 198:27-43. CrossRef Medline

Inokawa H, Yamada H, Matsumoto N, Muranishi M, Kimura M (2010) Juxtacellular labeling of tonically active neurons and phasically active neurons in the rat striatum. Neuroscience 168:395-404. CrossRef Medline

Joshua M, Adler A, Mitelman R, Vaadia E, Bergman H (2008) Midbrain dopaminergic neurons and striatal cholinergic interneurons encode the difference between reward and aversive events at different epochs of probabilistic classical conditioning trials. J Neurosci 28:1167311684. CrossRef Medline

Kimura M (1992) Behavioral modulation of sensory responses of primate putamen neurons. Brain Res 578:204-214. CrossRef Medline

Kimura M, Rajkowski J, Evarts E (1984) Tonically discharging putamen neurons exhibit set-dependent responses. Proc Natl Acad Sci U S A 81:4998-5001. CrossRef Medline

Koós T, Tepper JM (1999) Inhibitory control of neostriatal projection neurons by GABAergic interneurons. Nat Neurosci 2:467-472. CrossRef Medline

Lee IH, Seitz AR, Assad JA (2006) Activity of tonically active neurons in the monkey putamen during initiation and withholding of movement. J Neurophysiol 95:2391-2403. CrossRef Medline

Morris G, Arkadir D, Nevet A, Vaadia E, Bergman H (2004) Coincident but distinct messages of midbrain dopamine and striatal tonically active neurons. Neuron 43:133-143. CrossRef Medline

Ravel S, Sardo P, Legallet E, Apicella P (2001) Reward unpredictability inside and outside of a task context as a determinant of the responses of tonically active neurons in the monkey striatum. J Neurosci 21:57305739. Medline

Schulz JM, Reynolds JN (2013) Pause and rebound: sensory control of cholinergic signaling in the striatum. Trends Neurosci 36:41-50. CrossRef Medline

Sullivan MA, Chen H, Morikawa H (2008) Recurrent inhibitory network among striatal cholinergic interneurons. J Neurosci 28:8682-8690. CrossRef Medline

Threlfell S, Lalic T, Platt NJ, Jennings KA, Deisseroth K, Cragg SJ (2012) Striatal dopamine release is triggered by synchronized activity in cholinergic interneurons. Neuron 75:58-64. CrossRef Medline

Wang Z, Kai L, Day M, Ronesi J, Yin HH, Ding J, Tkatch T, Lovinger DM, Surmeier DJ (2006) Dopaminergic control of corticostriatal long-term synaptic depression in medium spiny neurons is mediated by cholinergic interneurons. Neuron 50:443-452. CrossRef Medline

Witten IB, Lin SC, Brodsky M, Prakash R, Diester I, Anikeeva P, Gradinaru V, Ramakrishnan C, Deisseroth K (2010) Cholinergic interneurons control local circuit activity and cocaine conditioning. Science 330:16771681. CrossRef Medline

Yamin HG, Stern EA, Cohen D (2013) Parallel processing of environmental recognition and locomotion in the mouse striatum. J Neurosci 33:473484. CrossRef Medline

Yarom O, Cohen D (2011) Putative cholinergic interneurons in the ventral and dorsal regions of the striatum have distinct roles in a two choice alternative association task. Front Syst Neurosci 5:36. CrossRef Medline 